\author{
Professor Irada DZHALLADOVA \\ E-mail: idzhalladova@gmail.com \\ Kyiv National Economic University, Kyiv, Ukraine \\ Associate Professor Stanislav ŠKAPA \\ E-mail: skapa@fbm.vutbr.cz \\ Brno University of Technology, Czech Republic \\ Senior Lecturer Veronika NOVOTNÁ \\ E-mail: novotna@fbm.vutbr.cz \\ Brno University of Technology, Czech Republic \\ Lecturer Alexandra BABYNYUK \\ E-mail: a.babynuk@gmail.com \\ Kyiv National Economic University, Kyiv, Ukraine
}

\title{
DESIGN AND ANALYSIS OF A MODEL FOR DETECTION OF INFORMATION ATTACKS IN COMPUTER NETWORKS
}

Abstract. Information technology has taken the world by storm. Its emergence has given rise to a new level of digital knowledge systems. Its application has been catalytic to the rapid changes taking place in the way people work, live and think, and is facilitating the development of our society and civilization in a new era. The current growing size and complexity of local computer networks also bring increasing demands for continuous monitoring of their proper performance, which is a prerequisite for their good efficiency, safety and reliability.

The aim of this paper is to introduce a cyberattack detection model based on a system of delay nonlinear differential equations and find its equilibrium state. The proposed model is based on a system of non-linear differential equations with delay and allows us to obtain a qualitative portrait of dynamic systems using the general Poincaré-Lyapunov theory based on the knowledge of the existence of stationary points and cycles and their mutual disposition. simulation.

Keywords: modelling, cyberattack detection, equilibrium, computer

JEL Classification: L86, C02

1. Introduction

The Internet and its widespread deployment in the last decade of the past century can undoubtedly be considered a revolution not only in the field of

DOI: $10.24818 / 18423264 / 53.3 .19 .06$ 
Irada Dzhalladova, Stanislav Škapa, Veronika Novotná, Alexandra Babynyuk

communication technologies, but also in the whole society and its lifestyle. Its use in everyday life has gained, and continues to gain, unprecedented proportions. The extent to which this phenomenon significantly interferes with our day-to-day activities opens enormous space for new threats it brings. The more dependent the functioning of the society is on the Internet, the more topical and serious these threats become. At present, almost all organizations process and store more and more information about their activities in digital form. At the same time, they use computers and data networks to interconnect not only within the organization, but also with their business contacts, customers, the state administration, etc.

However, with the rapid development of modern information system technologies, the possibility of their misuse increases. Almost every day we encounter cybercrime, data misuse, electronic theft and fraud. All of this highlights the need for adequate security, as negligence could lead to unprecedented consequences. As a result, it is no longer enough to implement cyber security measures on the physical perimeter of the organization. Every organization, from the smallest to the largest, should analyze the cyber risks it faces and take measures to minimize them. In this context, it is not only a risk of data loss as a result of a cyberattack, although it is still significant. The risks may include, for example, damage to brand reputation, logistics chain breakdown, financial loss or decommissioning of the infrastructure necessary for the organization to operate.

The aim of this paper is to introduce a cyberattack detection model based on a system of delay nonlinear differential equations, and find its equilibrium state. The behavior of the model is presented using computer simulation, and the Maple system is used for graphic representation of the results.

\section{Literature Search}

But besides the positive effect on society's development, ICT expansion has a negative effect, which is reflected in a relatively rapid increase in cyber risks, causing damage to individual companies as well as to society as a whole. This issue is addressed by Semenova (2017), both at the microeconomic as well as the macroeconomic level. Industrial control systems used in various industries are very sensitive to cyberattacks. As an example, we can mention a cyberattack against a German steel mill (Kovacs, 2014), or the destruction of nearly eleven nuclear facilities in Iran (Kelley, 2013). A paper by Razzaq (2013) focuses on threats to cyber security. The authors point out that the exponential growth in the use of cyberspace also exponentially increases cybercrime. They also note that the growing comprehensiveness and complexity of Web applications increase the number of design defects (it is said that up to $90 \%$ of web applications have a design defect), which allows for their misuse by cyber criminals.

Intrusion detection systems monitor network traffic formalicious activity. However, sophisticated cyber-attacks are often not discovered, because it is very 
Design and Analysis of a Model for Detection of Information Attacks in Computer Networks

difficult to distinguish them from normal operating deviations. Various ways to refine IDS's performance, such as neural networks, vector machines, "Naïve Bayes" algorithms (Abd-Elday, 2014) have been progressively applied. Škrjanc(2018) has introduced a new, evolving, Cauchy clustering (eCauchy) method in his paper. This method serves as the basis for extensive analyzing of cyber-attacks. The privacy and security features of the "internet of things" and the proposal for comprehensive security management can be found in a publication by Erfani (2017). Apaper by Wenbing (2018) addresses the problem of the sampled-data consensus problem for a group of nonlinear multiagent systems that are objects of cyber-attacks. Contradiction methods are applied to solve differential equations and to derive conditions under which systems are vulnerable to attacks. Nguen (2017) introduces a model inspired by epidemiologic models of virus propagation to derive an approximate solution to differential equations defining the Susceptible-Infected-Removed (SIR) model, and uses it to find an adequate defense against cyber-attacks.

An extensive analysis of the existing literature on cyber security has been conducted in a paper by Bordoff (2017) in which the authors synthesized areas of: (1) various types of cyber-attacks, (2) contributing factors related to cybersecurity behavior, and (3) strategies to improve cybersecurity behavior. In a paper by Ahmed (2014), the authors propose a method by which security requirements can be applied to business processes through collaboration between business and security analysts. A paper by Patterson (2013) is dedicated to optimizing investments in cyber security in critical infrastructure. The authors point out that these investments must be offset by savings resulting from intrusion prevention, cyber-attacks detection, and mitigation of physical damage caused by attackers on computer-controlled devices. Mukhopadhyay presents models to help businesses decide which product to choose for cyber security and the extent to which they can use it. The authors propose to choose the Copulaaided Bayesian Belief Network (CBBN) for the vulnerability assessment and expected loss calculation. Patte-Cornell (2018) focuses on assessing the risk of cyber-attacks in connection with organization budgeting taking into account the cost of protection. Cybercrime in Belgium is described in a paper byChen (2017) that at the same time verifies the hypothesis that organizations that have implemented cyber security policies have demonstrably lower losses resulting from security incidents.

\section{Description of the Model}

Information attacks on computer networks are a problem where the threat level increases every day. The "WannaCry" virus can be mentioned as an example of computer attacks in 2017, which infected more than 300,000 computers worldwide, due to the vulnerability of computers with older versions of Windows. Despite the virus being located and removed within a few days, its activities seriously damaged information systems of hospitals, banks and private companies. Another attack was the "NotPetya" virus, which encrypted user data without being able to recover it. 
Irada Dzhalladova, Stanislav Škapa, Veronika Novotná, Alexandra Babynyuk

An immediate response from cyber-attack specialists makes it possible to identify the weaknesses of computer networks that are then protected by programs called "patches". However, these patches become available with some delay, and attackers have time to exploit the error, even though the operating system manufacturers and other software manufacturers know about them. This state of affairs is unsatisfactory both for private companies and their management, as well as for the management of state organizations which, as their official duty, have to handle important or secret information.

The problem of delayed responses to these incidents, associated with the protection of information resources in cyber security systems of the information space was described by Konovich (2015). In this paper, an analysis was performed using non-linear Hutchinson, Lotchi, Volterra and Aronson models that allows for more flexible ways of detection, processing, and elimination of cyber-security incidents, and for the assessment of the level of information security based on statistical data and qualitative estimates made using nonlinear delay models.

A paper by Semikina (2015) proposes a model for information protection against malicious codes in computer networks, with respect to the time update of antivirus databases and the time needed for infecting computers with viruses.

We can conclude that the detection of information attacks on computer networks as the direction of scientific research and practice has a long history. Among other things, these attacks were simulated using delay differential equation models and equations of moments (see (Dzhalladova, 2017), (Dzhalladova and Babynyuk,2013), .

In this paper, we are considering a dynamic system of "information attack computer networks" without feedback. The system has the following components: Client A, Server B, Client-Side User, and the local network to which the server is connected. This model is described by an interaction between Client A and Server B without feedback, and by the influence of the local network on a server with request flow $\Delta(t)$.

Using balance ratios, we can describe the dynamic system of requests and responses as two differential equations with:

$$
\left\{\begin{array}{l}
\dot{x}_{1}(t)=\lambda(t)-x_{1}(t) \mu_{1} g_{1}\left(x_{1}(t)\right) \\
\dot{x}_{2}(t)=x_{1}(t-\tau) \mu_{2} g_{2}\left(x_{1}(t-\tau)-x_{2}(t-\tau)\right)
\end{array}\right.
$$

where $\bar{x}^{T}(t)=\left(x_{1}(t), x_{2}(t), \ldots, x_{n}(t)\right)$ - is the vector of phase variables and their component is the number of "packets" that are at that moment $t \geq 0$ on the i-th node; $f(x(t)), g(x(t))$ - are usage features that describe the cost of converting a data stream for transmission over a computer network; $\lambda(t), \Delta(t)$ - are inbound data streams from external objects. 
Design and Analysis of a Model for Detection of Information Attacks in Computer Networks

It is assumed that the functions $f(x(t)), g(x(t))$ characterize the degree of utilization of the packets coming into the node for further transmission. If $x_{s}(t)$ packets are processed, the result of processing is $x_{s}(t) f(x(t))$ of output packets. The flow rate at the node is defined as the difference between input and output per unit of time.

Let $\lambda(t)>0$ be the request sent by User A (input); is the time delay of information transmission from $\mathrm{A}$ to $\mathrm{B} ; \mathrm{x}_{1}$ are packets in $\mathrm{A}$ to be sent to $\mathrm{B}$ (requests); $\mathrm{x}_{2}$ are packets in $\mathrm{B}$ to be sent by the server to the local network (responses); , $\mu_{1}, \mu_{2}$ is the bandwidth of the respective nodes "information attacks" and "computer networks"; $g(x)=\frac{1}{1+x}-$ is the packet utilization function.

In the proposed model (1), the first equation corresponds to the "User-Client" node, and the second to the "Server-User" node".

Let's examine the system (1). With the packet utilization function, the system is as follows:

$$
\left\{\begin{array}{l}
\dot{x}_{1}(t)=\lambda(t)-\frac{x_{1}(t) \mu_{1}}{1+x_{1}(t)} \\
\dot{x}_{2}(t)=\frac{x_{1}(t-\tau) \mu_{2}}{1+x_{1}(t-\tau)}-\frac{x_{2}(t)}{1+x_{2}(t)},
\end{array}\right.
$$

Using substitution, the first equation

$$
y(t)=1+x_{1}(t), \Lambda(t)=\lambda(t)-\mu_{1}
$$

is reduced to

$$
\dot{y}(t)=\Lambda(t)+\frac{\mu_{1}}{y(t)}
$$

which is not integrated in quadratures (it is reduced to Abel's equation of the first kind). We will continue with the application of a research method based on system linearization.

We convert the equation to:

$$
\left\{\begin{array}{l}
\dot{x}_{1}(t)=\lambda(t)-\mu_{1} x_{1}(t)+\frac{x_{1}^{2}(t) \mu_{1}}{1+x_{1}(t)} \\
\dot{x}_{2}(t)=\mu_{2} x_{1}(t-\tau)-x_{2}(t)-\left[\mu_{2} \frac{x_{1}^{2}(t-\tau)}{1+x_{1}(t-\tau)}-\frac{x_{2}^{2}(t)}{1+x_{2}(t)}\right]
\end{array}\right.
$$


Irada Dzhalladova, Stanislav Škapa, Veronika Novotná, Alexandra Babynyuk

\subsection{Linearization close to the coordinate origin.}

With near-zero requirements $\lambda(t)>0$, the system may be replaced by a linearized system:

$$
\left\{\begin{array}{l}
\dot{x}_{1}(t)=\lambda(t)-x_{1}(t) \mu_{1} \\
\dot{x}_{2}(t)=x_{1}(t-\tau) \mu_{2}-x_{2}(t)
\end{array} .\right.
$$

The resulting system is a linear non-homogeneous system with a weak delay that allows integration. The first equation of the system is linear non-homogeneous. Its solution is:

$$
x_{1}(t)=\left\{\begin{array}{l}
\varphi_{1}(t), \quad \text { прu } \quad-\tau \leq t<0, \\
\varphi_{1}(0) e^{-\mu_{1}(t)}+\int_{0}^{t} e^{-\mu_{1}(t-s)} \lambda(s) d s, \text { npu } t \geq 0,
\end{array}\right.
$$

where $\varphi_{1}(t)$ - is a continuous function that determines the initial conditions. Using this solution in the second equation of the system (2), the result will be:

$$
\dot{x}_{2}(t)=\left\{\begin{array}{l}
-x_{2}(t)+\mu_{2} \varphi_{1}(t-\tau), \quad \text { npu } \quad-\tau \leq t<0, \\
-x_{2}(t)+\mu_{2}\left[\varphi_{1}(0) e^{-\mu_{1}(t)}+\int_{0}^{t} e^{-\mu_{1}(t-s)} \lambda(s) d s\right], \quad n p u \quad t \geq 0
\end{array}\right.
$$

In each of the intervals, the resulting equation will be linear nonhomogeneous. Its solution will be as follows:

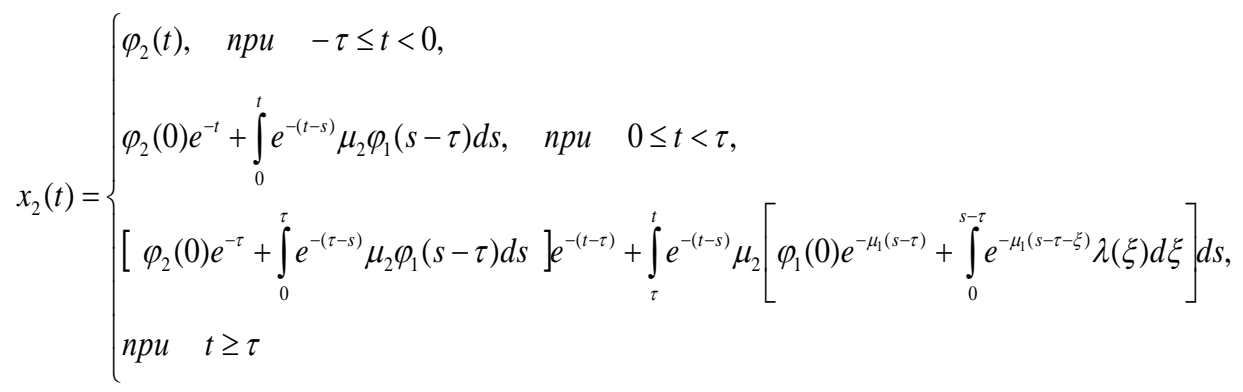


Design and Analysis of a Model for Detection of Information Attacks in Computer Networks

The solution of a linear non-homogeneous system with delay (1a), which satisfies the initial condition $x_{1}(t) \equiv \varphi_{1}(t), x_{2}(t) \equiv \varphi_{2}(t),-\tau \leq t<0$, where $\varphi_{1}(t)$, $\varphi_{2}(t)$ - are any continuous functions in the given interval, is as follows:

$$
x_{1}(t)=\left\{\begin{array}{l}
\varphi_{1}(t), \quad \text { прu } \quad-\tau \leq t<0, \\
\varphi_{1}(0) e^{-\mu_{1} t}+\int_{0}^{t} e^{-\mu_{1}(t-s)} \lambda(s) d s, \quad \text { npu } \quad t \geq 0,
\end{array}\right.
$$

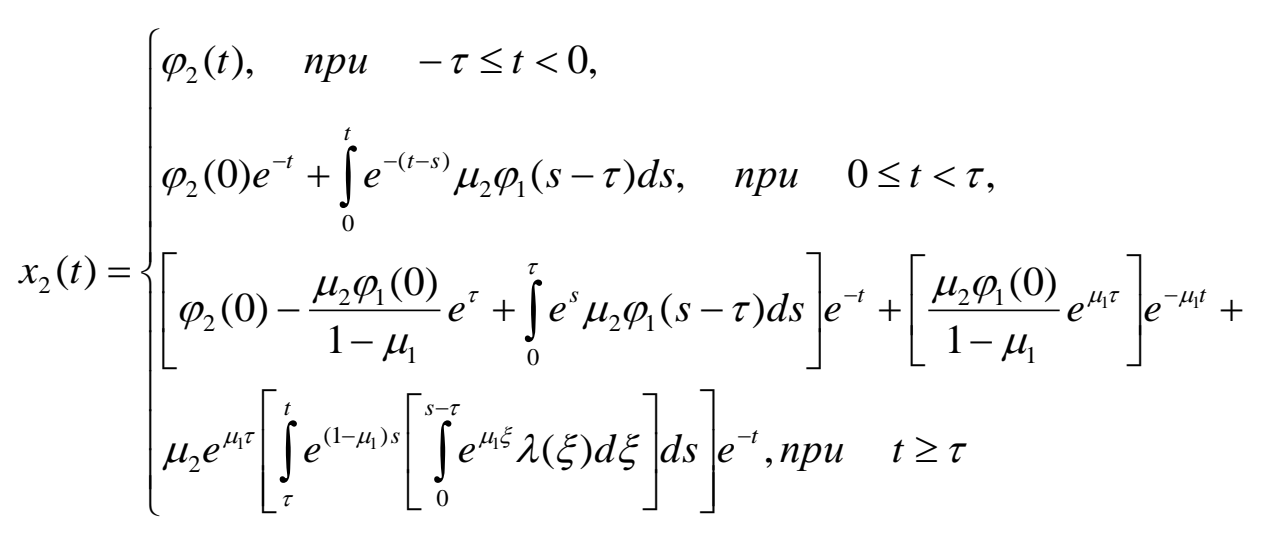

Let us investigate the dependence of the solution $x_{1}(t), x_{2}(t)$ on the size of the request $\lambda(t)$ and initial values. Let $\lambda(\mathrm{t})$ be a limited function, that is, that $|\lambda(t)| \leq \bar{\lambda}$. Functions $\varphi_{1}(t), \varphi_{2}(t) \quad$ are continuous and therefore limited. Let $\left|\varphi_{1}(t)\right| \leq \overline{\varphi_{1}},\left|\varphi_{2}(t)\right| \leq \overline{\varphi_{2}}$.

Taking into account the dependencies (5), the estimates for $x_{1}(t)$ and $x_{2}(t)$ are:

$$
\left|x_{1}(t)\right| \leq\left\{\begin{array}{l}
\overline{\varphi_{1}}, \quad \text { npu } \quad-\tau \leq t<0, \\
\left(\overline{\varphi_{1}}-\frac{\bar{\lambda}}{\mu_{1}}\right) e^{-\mu_{1} t}+\frac{\bar{\lambda}}{\mu_{1}}, \quad \text { npu } \quad t \geq 0
\end{array}\right.
$$


Irada Dzhalladova, Stanislav Škapa, Veronika Novotná, Alexandra Babynyuk

$$
\left|x_{2}(t)\right| \leq\left\{\begin{array}{l}
\overline{\varphi_{2}}, \quad n p u \quad-\tau \leq t<0, \\
\left(\overline{\varphi_{2}}-\mu_{2} \overline{\varphi_{1}}\right) e^{-t}+\mu_{2} \varphi_{1}, \quad \text { npu } \quad 0 \leq t<\tau, \\
\left(\overline{\varphi_{2}}-\mu_{2} \overline{\varphi_{1}}\right) e^{-t}+\frac{\mu_{2}}{1-\mu_{1}}\left(-\mu_{1} \overline{\varphi_{1}}+\bar{\lambda}\right) e^{-t+\tau}+\frac{\mu_{2}}{1-\mu_{1}}\left(\overline{\varphi_{1}}-\frac{\bar{\lambda}}{\mu_{1}}\right) e^{-\mu_{1}(t-\tau)}+ \\
\frac{\mu_{2}}{\mu_{1}}, n p u \quad t \geq \tau
\end{array}\right.
$$

Acquired dependencies are shown in graphs in Figure 1.

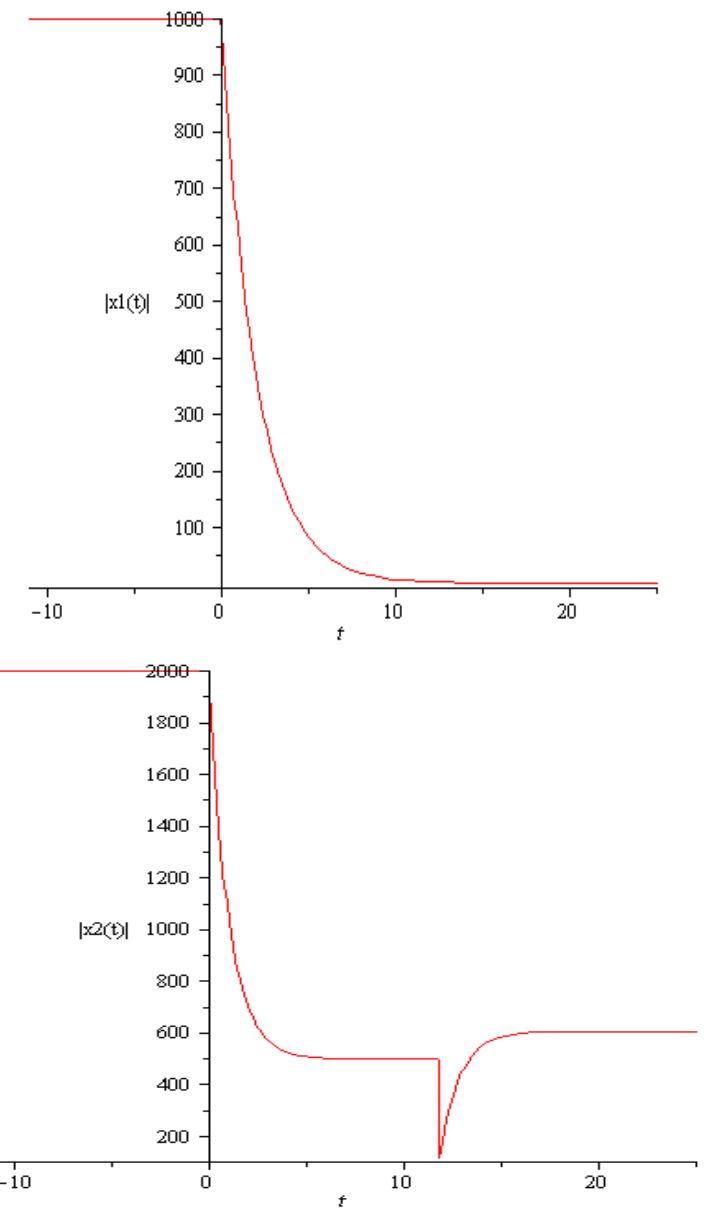

a)

b)

Figure 1. Graphic Interpretation of the Estimate for $x_{1}(t)$ (a) and $x_{2}(t)$ (b). 
Design and Analysis of a Model for Detection of Information Attacks in Computer Networks

\subsection{Linearization under the condition that the request is a constant value $\lambda$ (t) $\equiv$ const.}

As shown by the Poincare-Lyapunov theory, the qualitative phase portrait of the dynamic system, which is given by equation (2), is determined by special points and cycles. The special point $\left(x_{1}^{*}, x_{2}^{*}\right)$ is defined as the solution of the following system

$$
\left\{\begin{array}{l}
\lambda-\mu_{1} \frac{x_{1}^{*}}{1+x_{1}^{*}}=0 \\
\mu_{2} \frac{x_{1}^{*}}{1+x_{1}^{*}}-\frac{x_{2}^{*}}{1+x_{2}^{*}}=0
\end{array} .\right.
$$

From here we get:

$$
x_{1}^{*}=\frac{\lambda}{\mu_{1}-\lambda} \quad x_{2}^{*}=\frac{\lambda \mu_{2}}{\mu_{1}-\lambda \mu_{2}} \text {. }
$$

Let $\mu_{1} \neq \lambda, \quad \mu_{2} \neq \frac{\mu_{1}}{\lambda}$. We will perform linearization near the equilibrium position $\left(x_{1}^{*}, x_{2}^{*}\right)$. We get

$$
\left\{\begin{array}{l}
\dot{x}_{1}(t)=-\frac{\left(\mu_{1}-\lambda\right)^{2}}{\mu_{1}}\left[x_{1}(t)-\frac{\lambda}{\mu_{1}-\lambda}\right] \\
\dot{x}_{2}(t)=\frac{\mu_{2}}{\mu_{1}^{2}}\left(\mu_{1}-\lambda\right)^{2}\left[x_{1}(t-\tau)-\frac{\lambda}{\mu_{1}-\lambda}\right]-\left(\frac{\mu_{1}-\lambda \mu_{2}}{\mu_{1}}\right)^{2}\left[x_{2}(t)-\frac{\lambda \mu_{2}}{\mu_{1}-\lambda \mu_{2}}\right]
\end{array}\right.
$$

The characteristic equation of the resulting linearized system is as follows: 
Irada Dzhalladova, Stanislav Škapa, Veronika Novotná, Alexandra Babynyuk

$$
\left|\begin{array}{cc}
-\frac{\left(\mu_{1}-\lambda\right)^{2}}{\mu_{1}}-\lambda & 0 \\
e^{-\lambda \tau} \frac{\mu_{2}}{\mu_{1}^{2}}\left(\mu_{1}-\lambda\right)^{2} & -\left(\frac{\mu_{1}-\lambda \mu_{2}}{\mu_{1}}\right)^{2}-\lambda
\end{array}\right|=\left(\lambda+\frac{\left(\mu_{1}-\lambda\right)^{2}}{\mu_{1}}\right)\left(\lambda+\left(\frac{\mu_{1}-\lambda \mu_{2}}{\mu_{1}}\right)^{2}\right)=0
$$

Therefore, for any $\mu_{1}>0$ :

$$
\lambda_{1}=-\frac{\left(\mu_{1}-\lambda\right)^{2}}{\mu_{1}}<0, \quad \lambda_{2}=-\left(\frac{\mu_{1}-\lambda \mu_{2}}{\mu_{1}}\right)^{2}<0 \text {, }
$$

That is, the system has a weak delay and the special point is the point of "stable knot" type. Let us find the solution of the linear system in analytical form. Using notation (7), we can transcribe the system (6) as follows:

$$
\left\{\begin{array}{l}
\dot{x}_{1}(t)=\lambda_{1} x_{1}(t)+R_{1} \\
\dot{x}_{2}(t)=\lambda_{2} x_{2}(t)+r x_{1}(t-\tau)+R_{2}
\end{array}\right.
$$

where

$$
\begin{aligned}
& R_{1}=\frac{\lambda}{\mu_{1}}\left(\mu_{1}-\lambda\right) \quad R_{2}=\frac{\lambda^{2} \mu_{2}}{\mu_{1}^{2}}\left(1-\mu_{2}\right) \quad r=\frac{\mu_{2}}{\mu_{1}^{2}}\left(\mu_{1}-\lambda\right)^{2} \lambda_{1}=-\frac{\left(\mu_{1}-\lambda\right)^{2}}{\mu_{1}}, \\
& \lambda_{2}=-\left(\frac{\mu_{1}-\lambda \mu_{2}}{\mu_{1}}\right)^{2} .
\end{aligned}
$$

We integrate the first equation as follows:

$$
x_{1}(t)=\left\{\begin{array}{l}
\varphi_{1}(t), \quad n p u \quad-\tau \leq t<0, \\
\left(\varphi_{1}(0) e^{-\mu_{1} t}+\frac{R_{1}}{\lambda_{1}}\right) e^{\lambda_{1} t}-\frac{R_{1}}{\lambda_{1}}, \quad \text { npu } t>0
\end{array}\right.
$$

Now let's put the obtained solutions into the second equation 
Design and Analysis of a Model for Detection of Information Attacks in Computer Networks

$$
\dot{x}_{2}(t)=\left\{\begin{array}{l}
\lambda_{2} x_{2}(t)+R_{2}+r \varphi_{1}(t-\tau), \quad \text { npu } 0<t \leq \tau, \\
\lambda_{2} x_{2}(t)+R_{2}+r\left[\left(\varphi_{1}(0)+\frac{R_{1}}{\lambda_{1}}\right) e^{\lambda_{1}(t-\tau)}-\frac{R_{1}}{\lambda_{1}}\right], \quad \text { npu } t>\tau .
\end{array}\right.
$$

We have obtained first order linear non-homogeneous differential equations without delay. The solution is as follows:

$$
x_{2}(t)=\left\{\begin{array}{l}
\varphi_{2}(t), \quad n p u \quad-\tau \leq t<0, \\
\left(\varphi_{2}(0)+\frac{R_{2}}{\lambda_{2}}\right) e^{\lambda_{2} t}-\frac{R_{2}}{\lambda_{2}}+r \int_{0}^{t} e^{\lambda_{2}(t-s)} d s, \quad \text { npu } \quad 0 \leq t<\tau, \\
r\left[\varphi_{1}(0)-\frac{R_{1}}{\lambda_{1}}\right] \\
e^{\lambda_{1}(t-\tau)}+e_{2}^{\lambda_{2}(t-\tau)}\left[-\frac{r\left[\varphi_{1}(0)+\frac{R_{1}}{\lambda_{1}}\right]}{\lambda_{1}-\lambda_{2}}+\frac{r R_{1}}{\lambda_{1} \lambda_{2}}\right]+\left[\varphi_{1}(0)+\frac{R_{2}}{\lambda_{2}}\right] e^{\lambda_{2} t}+ \\
+\int_{0}^{\tau} e^{\lambda_{2}(t-s)} r \varphi_{1}(s-\tau) d s-\frac{R_{2}-r \frac{R_{1}}{\lambda_{1}}, n p u \quad t \geq \tau}{\lambda_{2}}, \text { in }
\end{array}\right.
$$

We have the solution to the system of equations.

For $t \rightarrow+\infty$ we get

$$
\begin{aligned}
& x_{1}(t) \rightarrow-\frac{R_{1}}{\lambda_{1}}=\frac{\lambda}{\mu_{1}-\lambda}=x_{1}^{*} \\
& x_{2}(t) \rightarrow-\frac{1}{\lambda_{2}}\left(R_{2}-r \frac{R_{1}}{\lambda_{1}}\right)=\frac{\lambda \mu_{2}}{\mu_{1}-\lambda \mu_{2}}=x_{2}^{*} .
\end{aligned}
$$

It follows that the equilibrium point $\left(x_{1}^{*}, x_{2}^{*}\right)$ is asymptotically stable.

Let's evaluate the dependence of solution values on inputs (request) and on system parameters. We have: 
Irada Dzhalladova, Stanislav Škapa, Veronika Novotná, Alexandra Babynyuk

$$
\begin{aligned}
& \left|x_{1}(t)\right| \leq\left\{\begin{array}{l}
\overline{\varphi_{1}}, \quad n p u \quad-\tau \leq t \leq 0, \\
\left(\overline{\varphi_{1}}+\frac{R_{1}}{\lambda_{1}}\right) e^{\lambda_{1} t}-\frac{R_{1}}{\lambda_{1}}, \quad n p u \quad t>0
\end{array}\right. \\
& \left|x_{2}(t)\right| \leq\left\{\begin{array}{l}
\overline{\varphi_{2}}, \quad n p u \quad-\tau \leq t \leq 0, \\
-\frac{R_{2}+r}{\lambda_{2}}+\left(\overline{\varphi_{2}}+\frac{R_{2}+r}{\lambda_{2}}\right) e^{\lambda_{2} t}, \quad \text { npu } \quad t>0
\end{array}\right.
\end{aligned}
$$

Acquired dependencies are shown in graphs in Figure 2
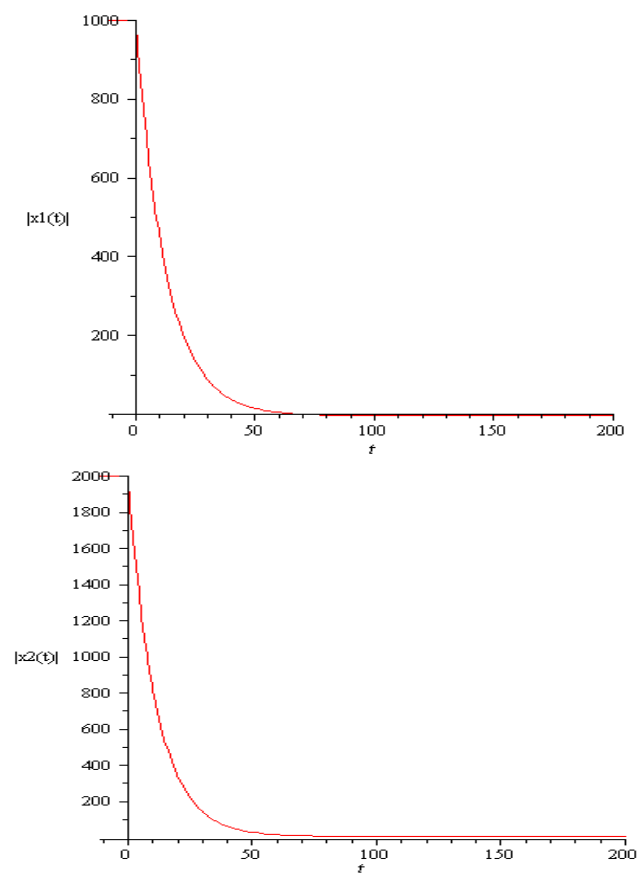

Figure 2. Graphic Interpretation of the Estimate for $x_{1}(t)$ (a) and $x_{2}(t)$ (b).

Note: Searching for the equilibrium position of the delay systems and their analysis is not as effective as with ordinary differential equations. This is caused by the fact that in the time-delay systems the exceptional point is stationary only if the 
Design and Analysis of a Model for Detection of Information Attacks in Computer Networks

trajectory stays in that point for a period not shorter than $\tau$. Otherwise, unlike ordinary equations, the trajectory "skips" over this point.

\section{Illustrative Example}

The development of science and technology in the beginning of the last century is closely related to the development of the so-called "Caratheodory" theory of differential equations, namely the ever-expanding theory of functional differential equations. In that period, mathematical modeling began to be used to describe real-life events. The theory dealing with the solution of the above and related problems can be studied, for example, in the monograph by (Kiguradze and Půža, 2003), dealing with linear problems. Application problems based on solutions of systems of delay differential equations can be found, for example in Bobalová and Maňásek (2007) and the literature cited therein.

Let's assume the following network parameters:

$$
\begin{gathered}
x_{1}(t)=15 * 10^{3} ; t<0 \\
x_{2}(t)=20 * 10^{3} ; t<0 \\
\mu_{1}=100 \\
\mu_{2}=200 \\
\tau=0.001
\end{gathered}
$$

We will construct the model in the interval $\langle 0 ; 60\rangle$ and we will observe the effect of the change of the function $\lambda(t)$.The solution is shown graphically.

\section{Example 1}

$$
\lambda(t)=5
$$

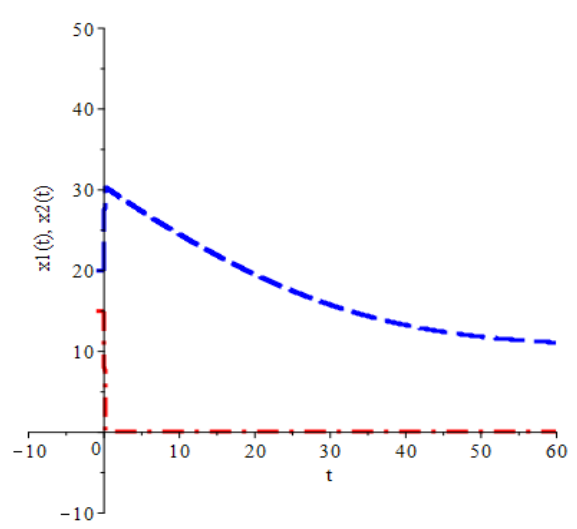

Fig. 3. Graphic Interpretation of Example 1 
Irada Dzhalladova, Stanislav Škapa, Veronika Novotná, Alexandra Babynyuk

\section{Example 2}

$$
\lambda(t)=5+\frac{\sin (t)}{0.1(t+0.001)}
$$

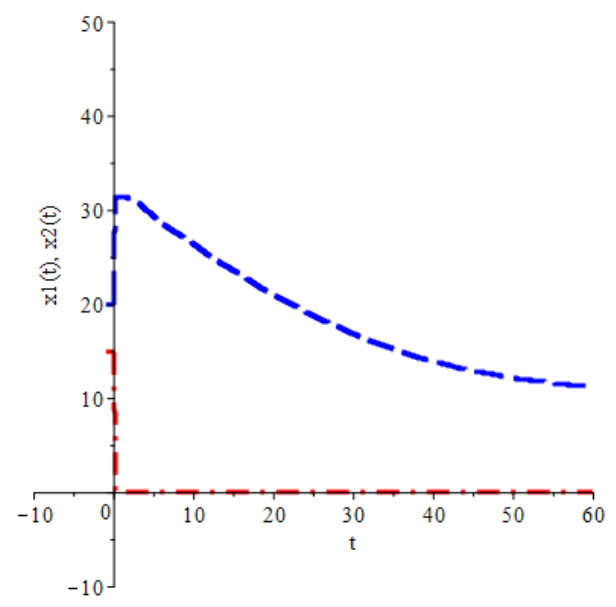

Fig. 4. Graphic Interpretation of Example 2

\section{Example 3}

$$
\lambda(t)=\left\{\begin{array}{c}
5+\frac{\sin (t)}{0.1(t+0.001)} t \in\langle 0 ; 20\rangle \\
25+\frac{\sin (t)}{0.1(t+0.001)} t \in(20 ; 35\rangle \\
15+\frac{\sin (t)}{0.1(t+0.001)} t \in(35 ; 60\rangle
\end{array}\right.
$$


Design and Analysis of a Model for Detection of Information Attacks in Computer Networks

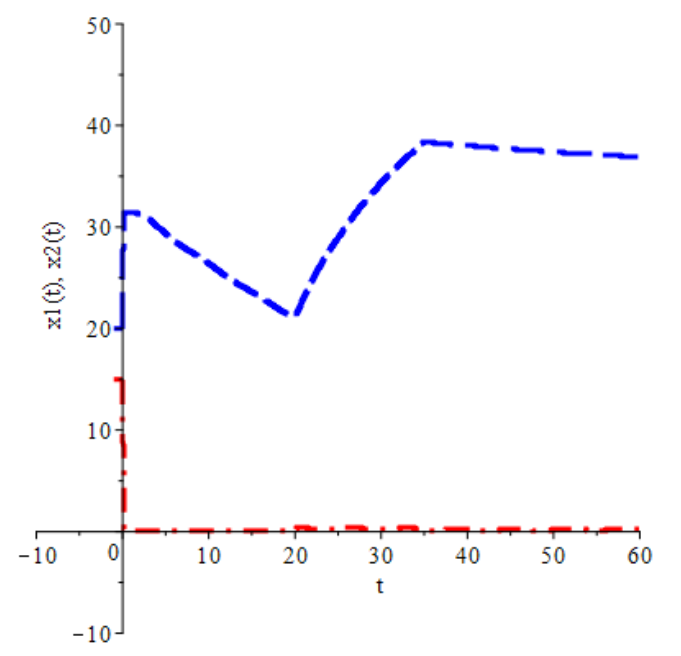

Fig. 5. Graphic Interpretation of Example 3

We can see that the function $\lambda(t)$ has a significant effect on the model solution. In a realistic situation, it can be expected that this function will not have the character of a constant, but that its values will fluctuate. The stepwise approximation method allows us to get solutions even for cases where the value of the function will change in jumps.

\section{Conclusion}

The current growing size and complexity of local computer networks also bring increasing demands for continuous monitoring of their proper performance, which is a prerequisite for their good efficiency, safety and reliability.

The proposed model is based on a system of non-linear differential equations with delay and allows us to obtain a qualitative portrait of dynamic systems using the general Poincaré-Lyapunov theory based on the knowledge of the existence of stationary points and cycles and their mutual disposition. When studying the nonlinear delay system that modeled the dynamics of the system "information attack - computer network ", the linearization method was used.

This was linearization of two types. First, linearization was performed close to the coordinate origin, which allowed obtaining solutions in an analytical form, and constructing the corresponding dependence on the initial values and the variable requirements $\lambda(\mathrm{t})$.

In the second approach, we suppose that the requirement has a constant value. As a result, the equilibrium position was found in the proximity of which linearization was performed. The applicability of this approach can be justified by the fact that the 
Irada Dzhalladova, Stanislav Škapa, Veronika Novotná, Alexandra Babynyuk

roots of the characteristic equation are negative. A linear stationary system was obtained and its general solution was constructed.

In conclusion, we can only say that today's cyberspace threats require model improvements, especially with the use of the existing and new mathematical tools (Dzhalladova and Babynyuk,2013), (Dzhalladova et all., 2013), (Novotna et all, 2016), (Khusainov, 2016).

\section{ACKNOWLEDGMENTS}

This paper was supported by grant FP-S-18-5524 "Information and Knowledge Management in the age of Industry 4.0"and GA 17-23448S "Modelling and simulation of sustainable investment decision-making”.

\section{REFERENCES}

[1] Abd-Eldayem, M. M. (2014), A Proposed HTTP Service Based IDS. Egyptian Informatics Journal, 15(1), 13-24. https://doi.org/10.1016/j.eij.2014.01.001;

[2] Ahmed, N. \&Matulevičius, R. (2014), Securing Business Processes Using Security Risk-Oriented Patterns; 36(4), 723-733; https://doi.org/10.1016/j.csi.2013.12.007;

[3]Bobalová, M. \&Maňásek, L. (2007), On Constructing a Solution of a Multipoint Boundary Value Problem. In CDDE 2006: Colloquium on differential and difference equations : [Brno, September 5-8, 2006] : Proceedings (pp. 93-100). Brno: Masaryk University; [4]Bordoff, S., Chen, Q. \& Yan, Z. (2017), Cyber Attacks, Contributing Factors, and Tackling Strategies. International Journal of Cyber Behavior, Psychology and Learning, 7(4), 68-82. https://doi.org/10.4018/IJCBPL.2017100106;

[5]Dzhalladova, I. A. \&Babynyuk, O. I. (2017), Computer Modeling of Threats to Elements of Cyberspace Using Delayed Differential Equations. In: Prospects of management activity of economic entities in the context of economic security: Materials of the international forum on security (pp. 219-221). Cherkasy: INFECO; [6]Dzhalladova, I. A. \&Babynyuk, O. I. (2017), Analytical Construction and Computer Realization of the Complex Labor Index for the Estimation of the Stability of the Labor Market with Undetermined Condition. In: Modeling of Management Processes in information economy (1st ed., pp. 92 - 105). Berdyansk;

[7]Dzhalladova, I. A., Baštinec, J., Diblík, J. \&Khusainov, D. Y. (2011), Estimates of Exponential Stability for Solutions of Stochastic Control Systems with Delay. Abstract and Applied Analysis, 2011(1), 1-14. https://doi.org/10.1155/2011/920412; 
Design and Analysis of a Model for Detection of Information Attacks in Computer Networks

[8]Dzhalladova, I. A. \&Khusainov, D. Y. (2013), Quadratic Systems with Delay. Journal of Automation and Information Sciences, 45(2), 13-23.

https://doi.org/10.1615/JAutomatInfScien.v45.i2.20;

[9]Dzhalladova, I., Khusainov, D. \&Kamratov, S. (2016), Investigation of

Solutions of Nonlinear Systems. Bulletin of the Taras Shevchenko National Taras

Shevchenko University. Cybernetics, 16(1), 46-54;

[10]Erfani, S., Ahmadi, M. \& Chen, L. (2017), The Internet of Things for Smart

Homes: An example. In: 2017- 8th Annual Industrial Automation and

Electromechanical Engineering Conference (IEMECON) (pp. 153-157). Bangkok:

IEEE. https://doi.org/10.1109/IEMECON.2017.8079580

[11]Chen, P., Visschers, J., Verstraete, C., Paoli, L., Huygens, C., Desmet, L. \&Joosen, W. (2017), The Relationship between the Cost of Cybercrime and Web Security Posture. In: Proceedings of the 11th European Conference on Software Architecture Companion Proceedings - ECSA '17 (pp. 115-120). New York, New York, USA: ACM Press. https://doi.org/10.1145/3129790.3129803;

[12]Kiguradze, I. \& Půža, B. (2003), Boundary Value Problems for Systems of Linear Functional Differential Equations (1st ed.). Brno: Masaryk University; [13]Kononovich, I. V., Majevskij, D. A. \&Podobnij, P. S. (2015), Models of System of the Cybersecurity Providing with Delay of Reaction on Incidents. Informatics \& Mathematical Methods In Simulation, 5(4), 339-346;

[14]Kovacs, E. (2014), Cyberattack on German Steel Plant Caused Significant Damage: Report [Online]. In: Security Week. Boston: Wired Business Media.

Retrieved from https://www.securityweek.com/cyberattack-german-steel-plant-causessignificant-damage-report;

[15]Limba, T., Plèta, T., Agafonov, K., Damkus, M. \&Tvaronavičienė, M. (2017), Cyber Security Management Model for Critical Infrastructure. Entrepreneurship and Sustainability Issues, 4(4), 559-573. https://doi.org/10.9770/jesi.2017.4.4(12);

[16]Mukhopadhyay, A., Chatterjee, S., Saha, D., Mahanti, A. \& Sadhukhan, S. K. (2013), Cyber-risk Decision Models: To Insure IT or not?. Decision Support Systems, 56(12), 11-26. https://doi.org/10.1016/j.dss.2013.04.004;

[17]Nguyen, B. (2017), Modelling Cyber Vulnerability Using Epidemic Models. In: SIMULTECH 2017 - Proceedings of the 7th International Conference on Simulation and Modeling Methodologies, Technologies and Applications 2017 (pp. 232-239). Madrid: SciTePress;

[18]Novotna, V., Půža, B., Dzhalladova, I. \&Khusainov, D. (2016), Effect of the After Effects on the Estimation of the Convergence of Solutions in Nonlinear Systems. Bulletin Of The Taras Shevchenko National Taras Shevchenko University. Cybernetics, 16(1), 27-31; 
Irada Dzhalladova, Stanislav Škapa, Veronika Novotná, Alexandra Babynyuk

[19]Paté-Cornell, M. -E., Kuypers, M., Smith, M. \& Keller, P. (2018), Cyber Risk Management for Critical Infrastructure: A Risk Analysis Model and Three Case Studies. Risk Analysis, 38(2), 226-241. https://doi.org/10.1111/risa.12844; [20]Patterson, I., Nutaro, J., Allgood, G., Kuruganti, T. \& Fugate, D. (2013), Optimizing Investments in Cyber-Security for Critical Infrastructure. In:

Proceedings of the Eighth Annual Cyber Security and Information Intelligence Research Workshop on - CSIIRW'13 (p. 1-). New York, New York, USA: ACM Press. https://doi.org/10.1145/2459976.2459999;

[21]Razzaq, A., Hur, A., Ahmad, H. F. \& Masood, M. (2013), Cyber Security: Threats, Reasons, Challenges, Methodologies and State of the Art Solutions for Industrial Applications. In: 2013 IEEE Eleventh International Symposium on Autonomous Decentralized Systems (ISADS) (pp. 1-6). IEEE.

https://doi.org/10.1109/ISADS.2013.6513420;

[22]Semenova, K. D. \&Tarasova, K. I. (2017), Establishment of the New Digital World and Issues of Cyber-Risks Management. Marketing and Management of Innovations, 2017(3), 236-244. https://doi.org/10.21272/mmi.2017.3-22;

[23]Semykina, N. A. (2014), Mathematical Model of Network Security on Virus with Delay. Modern Problems of Science and Education, 2014(9), 1982-1987;

[24]Škkrjanc, I., Ozawa, S., Ban, T. \&Dovžan, D. (2018), Large-Scale Cyber Attacks Monitoring Using Evolving Cauchy Possibilistic Clustering. Applied Soft Computing, 62(1), 592-601. https://doi.org/10.1016/j.asoc.2017.11.008;

[25]Zhang, W., Wang, Z., Liu, Y., Ding, D. \&Alsaadi, F. E. (2018), Sampled-Data Consensus of Nonlinear Multiagent Systems Subject to Cyber Attacks. International Journal Of Robust And Nonlinear Control, 28(1), 53-67.

https://doi.org/10.1002/rnc.3855. 